\title{
AVALIAÇÃO DE GENÓTIPOS DE BANANEIRA SOB IRRIGAÇÃO ${ }^{1}$
}

\author{
MARIA GERALDA VILELA RODRIGUES², ROSILENE FERREIRA SOUTO \\ SEBASTIÃO DE OLIVEIRA E SILVA ${ }^{4}$
}

\begin{abstract}
RESUMO - Como alternativa de genótipos de bananeira para o semi-árido irrigado do norte de Minas Gerais, foram avaliados quatro ciclos de produção da variedade Caipira (AAA) e dos híbridos SH3640 (AAAB), FHIA 1 (AAAB), FHIA 18 (AAAB) e Pioneira (AAAB), além de três ciclos da variedade Prata-Anã (AAB), no espaçamento $3 \mathrm{~m}$ x 2,7 m, em delineamento inteiramente casualizado, com 25 repetições de uma planta. Foram avaliados caracteres da planta e da produção, levando à conclusão de que os genótipos 'SH3640', 'FHIA 1' e 'FHIA 18' são mais produtivos que a 'Pioneira', 'Caipira' e 'Prata-Anã'. A 'Pioneira' apresentou maior crescimento inicial, porém, na floração, a maior altura foi apresentada pela 'Caipira', sendo que todos os genótipos se igualaram no quarto ciclo. A 'Caipira' apresentou maior número de frutos e a 'SH3640' apresentou cachos mais pesados e frutos maiores.
\end{abstract}

Termos para indexação: Musa sp., híbridos, variedades.

\section{EVALUATION OF GENOTYPES OF BANANA TREE UNDER IRRIGATION SYSTEM}

\begin{abstract}
As an alternative of banana tree genotypes for the irrigated semi-arid in the north of Minas Gerais, four production cycles of Caipira (AAA) variety and the hybrids SH3640 (AAAB), FHIA 1 (AAAB), FHIA 18 (AAAB) and Pioneira (AAAB) were evaluated, besides three cycles of the variety Prata-Anã (AAB), in the spacing $3 \mathrm{~m}$ x $2.7 \mathrm{~m}$, in complete randomized design, with 25 replicates of a plant. Plant and production characters were appraised, concluding that the genotypes 'SH3640', 'FHIA 1' and 'FHIA 18' are more productive than 'Pioneira', 'Caipira' and 'Prata-Anã'. 'Pioneira' presented higher initial growth, however, in the flowering stage, the largest height was presented by the 'Caipira', and all the genotypes were equaled in the fourth cycle. The 'Caipira' presented larger number of fruits and the 'SH3640' presented heavier bunches and bigger fruits.
\end{abstract}

Index Terms: Musa sp, hybrids, variety.

\section{INTRODUÇÃO}

Em 2004, o Brasil produziu 6.703 mil toneladas de bananas em 486 mil ha, resultando em um rendimento médio de 13,5 t ha(FAO, 2006). Há produção em todos os estados brasileiros, mas os cinco principais produtores foram, em 2004: São Paulo, Bahia, Santa Catarina, Minas Gerais e Pará, produzindo 1.090; 760; 6667; 550 e 540 mil toneladas, com produtividade de 20,7; 13,9; 22,0;14,6 e 12,8 $\mathrm{tha}^{-1}$, respectivamente (Agrianual, 2006). Portanto, apesar de ser o segundo maior produtor mundial, a produtividade de banana no Brasil é baixa, devido ao baixo nível tecnológico adotado e à utilização de variedades pouco produtivas, além de suscetíveis a diversas pragas. Em Minas Gerais, a preferência do consumidor é pelo subgrupo Prata.

As pragas provocam severas perdas na produção de banana, que, a depender das circunstâncias, podem ser de até $100 \%$. Os fungos constituem os maiores problemas da bananicultura mundial, causando doenças, como a murcha de Fusarium (mal-doPanamá) e as manchas foliares (sigatoka amarela e negra). Uma das estratégias para a solução desses problemas é a criação de novas variedades produtivas, resistentes às pragas e doenças, com porte $\mathrm{e}$ ciclo reduzidos e frutos de qualidade (Silva et al., 2001).

Este trabalho foi conduzido para avaliar variedades e híbridos da 'Prata', cultivados sob irrigação no norte de Minas, quanto ao porte, ciclo, produção e qualidade da produção.

\section{MATERIAL E MÉTODOS}

O trabalho foi conduzido em área da Epamig/CTNM, localizada no Sistema III da Área F do Projeto Jaíba, no período de 1997 a 2000. O solo da área foi classificado como Latossolo VermelhoAmarelo, de classe textural Franco-Argilo-Arenosa $\left(680 \mathrm{~g} \mathrm{~kg}^{-1} \mathrm{de}\right.$ areia, $220 \mathrm{~g} \mathrm{~kg}^{-1}$ de argila e $100 \mathrm{~g} \mathrm{~kg}^{-1}$ de silte). O clima da região é Aw, segundo a classificação de Köppen, com precipitação anual média de $750 \mathrm{~mm}$, o que torna a irrigação dos bananais uma prática obrigatória.

Foram avaliados quatro ciclos de produção da variedade Caipira (AAA); dos híbridos de 'Prata-Anã': SH3640 (AAAB), FHIA 1 (AAAB), FHIA 18 (AAAB) e Pioneira (AAAB); além de três ciclos da variedade Prata-Anã (AAB). Avaliaram-se 25 plantas de cada genótipo, distribuídas na área aleatoriamente (DIC). Utilizaram-se mudas provenientes de cultura de tecidos, plantadas no espaçamento de 3,0 m x 2,7m, sob microaspersão. Por falta de um sistema produtivo específico para estes materiais, as plantas foram conduzidas segundo recomendações feitas para a 'Prata-Anã' cultivada na região (Silva et al., 1999), com adubação mensal e irrigação diária. Não se efetuou controle de Sigatoka amarela.

Os caracteres avaliados foram altura da planta $(\mathrm{cm})$; perímetro do pseudocaule a $30 \mathrm{~cm}$ do solo $(\mathrm{cm})$; número de folhas vivas aos três $(3 \mathrm{M})$ e seis $(6 \mathrm{M})$ meses pós-plantio e na floração do primeiro (FL C1), segundo (FL C2), terceiro (FL C3) e quarto ciclos (FL C4); número de folhas vivas no momento da colheita do primeiro (COL C1), segundo (COL C2), terceiro (COL C3) e quarto ciclos (COL $\mathrm{C} 4)$; dias entre o plantio e a colheita do primeiro ciclo e período, em dias, entre a floração e a colheita do primeiro $(\mathrm{C} 1)$, segundo $(\mathrm{C} 2)$, terceiro (C3) e quarto ciclos (C4). A produção de cada um dos quatro ciclos foi caracterizada com massa de frutos por cacho $(\mathrm{kg})$; número de frutos por cacho; massa média dos frutos $(\mathrm{g})$; número de pencas por cacho; comprimento $(\mathrm{cm})$, diâmetro $(\mathrm{cm})$, massa $(\mathrm{g})$ do fruto e espessura da casca $(\mathrm{cm})$ do fruto central da mão externa da segunda penca; massa $(\mathrm{kg})$ e número de frutos de cada uma das cinco primeiras pencas do cacho. Calcularam-se a massa média de frutos por cacho e a produtividade $\left(\mathrm{t} \mathrm{ha}^{-1} \mathrm{ano}^{-1}\right)$, considerando os quatro ciclos. Os dados foram submetidos à análise estatística, e as médias dos tratamentos, comparadas pelo teste de Tukey a 5\%.

\footnotetext{
${ }^{1}$ (Trabalho 183-2005). Recebido: 07-11-2005. Aceito para publicação:06-10-2006. 1 Projeto financiado pelo consórcio de pesquisa Epamig/Embrapa/ Codevasf/BIRD

${ }^{2}$ Eng. Agrônoma, D.Sc., Pesquisadora Epamig/CTNM, Nova Porteirinha-MG, Cx. P. 12, CEP 39527.000, magevr@epamig.br

${ }_{3}^{3}$ Eng. Agrônoma, D. Sc., Ministério da Agricultura, Goiânia-GO, rosilenef@ agricultura.gov.br

${ }^{4}$ Eng. Agrônomo, D. Sc., Pesquisador Embrapa, Centro Nacional de Pesquisa em Mandioca e Fruticultura, Cruz das Almas-BA, Cx. P. 007, CEP 44380.000, ssilva@cnpmf.embrapa.br
} 


\section{RESULTADOS E DISCUSSÃO}

A 'Pioneira' apresentou maior crescimento inicial, com maior altura e perímetro de pseudocaule aos três meses pós-plantio, e, assim como 'SH3640' e 'FHIA 1', aos seis meses (Tabela 1). A 'Pioneira' apresentou maior número de folhas vivas ao três e seis meses pósplantio. No momento da floração, entretanto, o maior porte foi apresentado pela 'Caipira', igualando-se à 'SH3640' no segundo ciclo, não havendo diferença entre os genótipos no quarto ciclo. Apesar de mais alta, a 'Caipira' apresentou o menor perímetro de pseudocaule, com exceção do primeiro ciclo, onde a 'Pioneira' apresentou pseudocaule com menor perímetro. No primeiro ciclo, as plantas apresentaram altura próxima de $3 \mathrm{~m}$, concordando com Silva et al. (2000), porém, nos ciclos seguintes, todas superaram esse valor. Todos os genótipos avaliados apresentaram incremento na altura e no perímetro do pseudocaule ao longo dos ciclos. Esse ganho foi constante para 'Pioneira' e estabilizou no terceiro ciclo para os outros genótipos, exceto 'Prata-Anã' que, para essas características, só foi avaliada nos dois primeiros ciclos. Segundo Silva et al. (2003), avaliando dois ciclos de produção de genótipos de bananeira em diversas localidades, esse caráter normalmente só se estabiliza em gerações mais avançadas.

Não houve constância no número de folhas vivas (NFV), no momento da colheita, entre os ciclos (Tabela 1). Nos dois primeiros ciclos, o maior valor foi apresentado pela 'Prata-Anã' e, nos seguintes, quando a 'Prata-Anã' não foi avaliada, pela 'Caipira', 'Pioneira' e 'SH3640' no terceiro, e por todas, exceto 'Caipira', no quarto ciclo. Tão importante quanto um número adequado de folhas, no momento da floração, é que a planta consiga manter as folhas durante o período de enchimento do cacho, já que não há emissão foliar após a floração. Não foi observada diferença entre os genótipos, no primeiro ciclo, para esta característica. A 'Prata-Anã' apresentou maior número de folhas vivas no segundo ciclo e o menor no terceiro ciclo, quando se igualou às outras, com exceção da 'SH3640'. No quarto ciclo, o maior número de folhas vivas na colheita foi apresentado pela 'SH3640'. Todos os genótipos apresentaram mais de 12 folhas vivas na floração e mais de sete no momento da colheita, nos quatro ciclos avaliados.
Esses valores são superiores aos observados por SILVA et al. (2000). Os genótipos mais precoces foram 'Pioneira' e 'Prata-Anã' (Tabela 2). O menor tempo de permanência do cacho na planta (dias entre a floração e a colheita) foi apresentado pela 'Caipira', SH3640', 'FHIA 1' e 'FHIA 18' no primeiro e segundo ciclos; 'Caipira', SH3640' e 'FHIA 18' no terceiro ciclo; 'SH3640' e 'FHIA 18' no quarto ciclo. A precocidade dita retorno econômico mais rápido ao produtor, enquanto o menor tempo de permanência na planta reduz o tempo de exposição do cacho a agentes causadores de danos.

A massa de frutos por cacho aumentou até o quarto ciclo para 'Pioneira' e 'SH3640', porém houve redução no quarto ciclo em relação ao terceiro, para 'FHIA 1' e 'FHIA 18' (Tabela 4). A 'Caipira' apresentou os maiores valores no primeiro e quarto ciclos. Os cachos mais pesados foram da 'SH3640' e 'FHIA 1' no primeiro e segundo ciclos; 'SH3640', 'FHIA 1' e 'FHIA 18' no terceiro ciclo; e 'SH3640' no quarto ciclo. Portanto a 'SH3640' se destacou nos quatro ciclos avaliados, quanto à massa de frutos por cacho.

A 'Pioneira' apresentou massa de frutos por cacho semelhante à da 'Prata-Anã' nos três primeiros ciclos. 'SH3640', 'FHIA 1' e 'FHIA 18' apresentaram, respectivamente, cachos 2; 1,9 e 1,5 vezes mais pesados que os da 'Prata-Anã' nos dois primeiros ciclos e 2,5; 2,6 e 2,3 vezes superiores no terceiro ciclo. Silva et al. (2003), avaliando, entre outros, os dois primeiros ciclos desse mesmo bananal, observaram que 'SH3640', 'FHIA 1' e 'FHIA 18' superaram a variedade genitora ('Prata-Anã'), e que a 'Pioneira' apresentou médias semelhantes à da 'Prata-Anã'. Aqui, portanto, observa-se que essa tendência se acentuou no ciclo seguinte. Quanto à massa média do cacho, nos quatro ciclos, os genótipos apresentaram a seguinte ordem decrescente: 'SH3640' > 'FHIA 1' > 'FHIA 18' > 'Caipira' = 'Pioneira'.

Também como observado por Silva et al. (2003), o número de frutos e de pencas por cacho não apresenta uma relação perfeita com a massa do cacho, uma vez que essa depende também do tamanho do fruto. A 'Caipira' apresentou maior número de frutos no primeiro ciclo; 'FHIA 1' e 'FHIA 18' no segundo e terceiro ciclos; 'Caipira', 'FHIA 1' e 'FHIA 18' no quarto ciclo (Tabela 4). O maior número de pencas foi apresentado pela 'FHIA 1' no primeiro ciclo;

TABELA 1- Médias dos caracteres altura da planta $(\mathrm{cm})$; perímetro do pseudocaule a $30 \mathrm{~cm}$ do solo $(\mathrm{cm})$; número de folhas vivas aos três (3M) e seis $(6 \mathrm{M})$ meses pós-plantio e na floração do primeiro (FL C1), segundo (FL C2), terceiro (FL C3) e quarto ciclos (FL C4); número de folhas vivas na colheita do primeiro (COL C1), segundo (COL C2), terceiro (COL C3) e quarto ciclos (COL C4), dos genótipos avaliados. Epamig/CTNM, Nova Porteirinha-MG.

\begin{tabular}{|c|c|c|c|c|c|c|c|c|}
\hline & & & & Genót & & & & \\
\hline & Época & Pioneira & Caipira & SH3640 & FHIA 1 & FHIA 18 & $\begin{array}{l}\text { Prata } \\
\text { Anã }\end{array}$ & $\begin{array}{l}\text { CV } \\
(\%)\end{array}$ \\
\hline Altura & $3 \mathrm{M}$ & $91 \mathrm{a}$ & $72 \mathrm{bc}$ & $68 \mathrm{bc}$ & $62 \mathrm{~cd}$ & $59 \mathrm{~d}$ & & 15,9 \\
\hline & $6 \mathrm{M}$ & $155 a$ & $140 \mathrm{~b}$ & $148 \mathrm{ab}$ & $143 \mathrm{ab}$ & $141 \mathrm{~b}$ & & 13,2 \\
\hline planta & FL C1 & $198 \mathrm{e}$ & 310 a & $290 \mathrm{~b}$ & $269 \mathrm{c}$ & $248 \mathrm{~d}$ & $248 \mathrm{~d}$ & 7,2 \\
\hline$(\mathrm{cm})$ & FL C2 & $278 \mathrm{~d}$ & 370 a & 387 a & $349 b$ & $325 \mathrm{c}$ & $324 c$ & 7,2 \\
\hline & FL C3 & $363 c$ & 448 a & $424 b$ & $420 \mathrm{~b}$ & $413 \mathrm{~b}$ & & 5,8 \\
\hline & FL C4 & $408 \mathrm{a}$ & 426 a & 422 a & $417 \mathrm{a}$ & $415 a$ & & 6,7 \\
\hline Perí- & $3 \mathrm{M}$ & $10 \mathrm{a}$ & $7 \mathrm{c}$ & $8 \mathrm{~b}$ & $8 \mathrm{~b}$ & $7 \mathrm{c}$ & & 14,3 \\
\hline metro & $6 \mathrm{M}$ & 49 a & $35 \mathrm{~b}$ & $48 \mathrm{a}$ & $46 a$ & $43 a$ & & 18,7 \\
\hline$(\mathrm{cm})$ & FL C1 & $59 \mathrm{~d}$ & $67 \mathrm{c}$ & 81 a & $80 \mathrm{a}$ & $73 b$ & $67 c$ & 7,4 \\
\hline & FL C2 & $70 \mathrm{~d}$ & $69 d$ & 98 a & $92 \mathrm{~b}$ & $85 \mathrm{c}$ & $84 c$ & 8,8 \\
\hline & FL C3 & $92 \mathrm{c}$ & $81 \mathrm{~d}$ & $123 \mathrm{a}$ & $113 \mathrm{~b}$ & $111 \mathrm{~b}$ & & 9,0 \\
\hline & FL C4 & $103 \mathrm{c}$ & $85 \mathrm{~d}$ & $124 \mathrm{a}$ & $113 \mathrm{~b}$ & $108 \mathrm{bc}$ & & 8,9 \\
\hline Número & $3 \mathrm{M}$ & $12 \mathrm{a}$ & $11 \mathrm{~d}$ & $11 \mathrm{~d}$ & $12 a b$ & $19 \mathrm{~cd}$ & & 8,6 \\
\hline de & $6 \mathrm{M}$ & $14 a$ & $12 \mathrm{~b}$ & $13 \mathrm{~b}$ & $13 \mathrm{~b}$ & $13 \mathrm{~b}$ & & 10,8 \\
\hline folhas & FL C1 & $16 \mathrm{~d}$ & $14 \mathrm{e}$ & $17 \mathrm{~cd}$ & $19 \mathrm{~b}$ & $18 \mathrm{bc}$ & $21 a$ & 10,4 \\
\hline vivas & FL C2 & $12 \mathrm{c}$ & $13 \mathrm{bc}$ & $15 \mathrm{~b}$ & $13 \mathrm{bc}$ & $12 \mathrm{c}$ & $17 a$ & 14,3 \\
\hline & FL C3 & $14 a$ & $12 a b$ & $14 a$ & $13 \mathrm{~b}$ & $13 \mathrm{~b}$ & & 13,2 \\
\hline & FL C4 & $13 a b$ & $12 \mathrm{~b}$ & $14 \mathrm{a}$ & $14 \mathrm{a}$ & $13 a$ & & 16,8 \\
\hline Número & $\mathrm{COL} \mathrm{C1}$ & $9 a$ & $8 a$ & $8 a$ & $8 \mathrm{a}$ & $8 a$ & $9 a$ & 22,0 \\
\hline de & COL C2 & $7 \mathrm{c}$ & $8 \mathrm{bc}$ & $9 \mathrm{~b}$ & $8 \mathrm{bc}$ & $8 \mathrm{bc}$ & $11 a$ & 20,3 \\
\hline folhas & COL C3 & $9 a b$ & $9 \mathrm{ab}$ & $11 \mathrm{a}$ & $9 a b$ & $9 a b$ & $8 \mathrm{~b}$ & 19,1 \\
\hline vivas & COL C4 & $9 \mathrm{~b}$ & $9 \mathrm{~b}$ & $11 \mathrm{a}$ & $8 \mathrm{~b}$ & $8 \mathrm{~b}$ & & 18,2 \\
\hline
\end{tabular}

Médias seguidas pela mesma letra na linha não diferem entre si, pelo teste de Tukey, a 5\%. 
TABELA 2- Período em dias, entre o plantio e a colheita do primeiro ciclo e entre a floração e a colheita do primeiro (C1), segundo (C2), terceiro (C3) e quarto ciclos (C4), dos genótipos avaliados. Epamig/CTNM, Nova Porteirinha-MG.

\begin{tabular}{|c|c|c|c|c|c|c|c|c|}
\hline \multirow[b]{2}{*}{ Dias } & \multirow[b]{2}{*}{ Ciclo } & \multicolumn{6}{|c|}{ Genótipos } & \multirow[b]{2}{*}{$\begin{array}{l}\text { CV } \\
(\%)\end{array}$} \\
\hline & & Pioneira & Caipira & SH3640 & FHIA 1 & FHIA 18 & $\begin{array}{c}\text { Prata } \\
\text { Anã }\end{array}$ & \\
\hline $\begin{array}{l}\text { Plantio- } \\
\text { colheita }\end{array}$ & $\mathrm{C1}$ & $310 \mathrm{c}$ & $382 \mathrm{a}$ & $370 \mathrm{a}$ & $347 b$ & $344 \mathrm{~b}$ & $320 \mathrm{c}$ & 4,5 \\
\hline $\begin{array}{l}\text { Floração- } \\
\text { colheita }\end{array}$ & $\begin{array}{l}\text { C 1 } \\
\text { C2 } \\
\text { C3 } \\
\text { C4 }\end{array}$ & $\begin{array}{l}131 \mathrm{~b} \\
126 \mathrm{~b} \\
149 \mathrm{a} \\
136 \mathrm{a}\end{array}$ & $\begin{array}{c}116 \mathrm{c} \\
119 \mathrm{bc} \\
122 \mathrm{~b} \\
128 \mathrm{a}\end{array}$ & $\begin{array}{c}118 \mathrm{c} \\
111 \mathrm{c} \\
115 \mathrm{~b} \\
112 \mathrm{ab}\end{array}$ & $\begin{array}{c}119 \mathrm{c} \\
115 \mathrm{bc} \\
145 \mathrm{a} \\
128 \mathrm{a}\end{array}$ & $\begin{array}{c}121 \mathrm{c} \\
114 \mathrm{bc} \\
129 \mathrm{ab} \\
108 \mathrm{~b}\end{array}$ & $\begin{array}{l}181 \mathrm{a} \\
151 \mathrm{a}\end{array}$ & $\begin{array}{c}7,4 \\
10,7 \\
18,7 \\
18,9\end{array}$ \\
\hline
\end{tabular}

Médias seguidas pela mesma letra na linha não diferem entre si, pelo teste de Tukey, a 5\%.

TABELA 3- Massa de frutos por cacho (kg), número de frutos por cacho e número de pencas por cacho, no primeiro (C1), segundo (C2), terceiro (C3) e quarto ciclos (C4), dos genótipos avaliados. Epamig/CTNM, Nova Porteirinha-MG.

\begin{tabular}{|c|c|c|c|c|c|c|c|c|}
\hline & \multirow[b]{2}{*}{ Ciclo } & \multicolumn{6}{|c|}{ Genótipos } & \multirow[b]{2}{*}{$\begin{array}{l}\text { CV } \\
(\%)\end{array}$} \\
\hline & & Pioneira & Caipira & SH3640 & FHIA 1 & FHIA 18 & $\begin{array}{l}\text { Prata } \\
\text { Anã }\end{array}$ & \\
\hline \multirow{4}{*}{$\begin{array}{l}\text { Número de } \\
\text { frutos por } \\
\text { cacho }\end{array}$} & C 1 & $96 \mathrm{e}$ & $181 \mathrm{a}$ & $138 \mathrm{c}$ & $156 \mathrm{~b}$ & $145 \mathrm{bc}$ & $119 \mathrm{~d}$ & 11,0 \\
\hline & $\mathrm{C} 2$ & $120 \mathrm{de}$ & $174 b$ & $155 \mathrm{c}$ & $193 a$ & $180 \mathrm{ab}$ & $134 d$ & 10,1 \\
\hline & $\mathrm{C} 3$ & $145 \mathrm{~d}$ & $208 \mathrm{~b}$ & $179 \mathrm{c}$ & $226 a b$ & $234 \mathrm{a}$ & $159 \mathrm{~d}$ & 10,7 \\
\hline & $\mathrm{C} 4$ & $163 \mathrm{c}$ & $238 \mathrm{a}$ & $183 \mathrm{~b}$ & $231 \mathrm{a}$ & $230 \mathrm{a}$ & & 11,5 \\
\hline \multirow{4}{*}{$\begin{array}{l}\text { Número de } \\
\text { pencas por } \\
\text { cacho }\end{array}$} & C 1 & $7,7 \mathrm{e}$ & $9,2 \mathrm{~cd}$ & $9,8 \mathrm{bc}$ & $10,7 \mathrm{a}$ & $9,9 \mathrm{~b}$ & $8,7 \mathrm{~d}$ & 8,3 \\
\hline & $\mathrm{C} 2$ & $8,8 \mathrm{~d}$ & $9,1 \mathrm{~cd}$ & $10,0 \mathrm{~b}$ & $11,6 \mathrm{a}$ & $11,3 \mathrm{a}$ & $9,6 \mathrm{bc}$ & 7,1 \\
\hline & C3 & $9,7 \mathrm{c}$ & $11,0 \mathrm{~b}$ & $10,1 \mathrm{bc}$ & 13,0 a & $13,1 \mathrm{a}$ & $10,4 \mathrm{bc}$ & 8,6 \\
\hline & $\mathrm{C} 4$ & $10,7 \mathrm{~b}$ & $10,8 \mathrm{~b}$ & $10,7 \mathrm{~b}$ & 13,1 a & $12,9 \mathrm{a}$ & & 8,1 \\
\hline \multirow{5}{*}{$\begin{array}{l}\text { Massa de } \\
\text { frutos por } \\
\text { cacho } \\
(\mathrm{kg})\end{array}$} & C 1 & $11,8 \mathrm{c}$ & $21,3 \mathrm{~b}$ & 29,2 a & $28,1 \mathrm{a}$ & $23,7 b$ & $13,9 \mathrm{c}$ & 17,9 \\
\hline & $\mathrm{C} 2$ & $16,4 \mathrm{c}$ & $16,1 \mathrm{c}$ & $36,2 \mathrm{a}$ & $34,0 \mathrm{ab}$ & $30,5 \mathrm{~b}$ & $18,8 \mathrm{c}$ & 16,0 \\
\hline & C3 & $19,4 \mathrm{~b}$ & $18,0 \mathrm{~b}$ & $50,6 \mathrm{a}$ & 51,6 a & $46,6 \mathrm{a}$ & $18,5 \mathrm{~b}$ & 22,5 \\
\hline & $\mathrm{C} 4$ & $25,5 \mathrm{c}$ & $22,7 \mathrm{c}$ & 54,5 a & $39,4 \mathrm{~b}$ & $35,4 \mathrm{~b}$ & & 21,4 \\
\hline & Média & $18,2 \mathrm{~d}$ & $19,5 \mathrm{~d}$ & $42,5 \mathrm{a}$ & $38,5 \mathrm{~b}$ & $34,2 \mathrm{c}$ & & 11,6 \\
\hline t ha ${ }^{-1}$ ano $^{-1}$ & Média & $40,86 \mathrm{~b}$ & $41,62 b$ & $70,70 \mathrm{a}$ & $74,83 \mathrm{a}$ & $72,47 \mathrm{a}$ & & 12,00 \\
\hline
\end{tabular}

Médias seguidas pela mesma letra na linha não diferem entre si, pelo teste de Tukey, a 5\%.

TABELA 4- Massa média dos frutos, massa, comprimento, diâmetro e espessura de casca do fruto central da mão externa da segunda penca, no primeiro (C1), segundo (C2), terceiro (C3) e quarto ciclos (C4), dos genótipos avaliados. Epamig/CTNM, Nova PorteirinhaMG.

\begin{tabular}{|c|c|c|c|c|c|c|c|c|}
\hline & \multirow[b]{2}{*}{ Ciclo } & \multicolumn{6}{|c|}{ Genótipos } & \multirow[b]{2}{*}{$\begin{array}{l}\text { CV } \\
(\%)\end{array}$} \\
\hline & & Pioneira & Caipira & SH3640 & FHIA 1 & FHIA 18 & $\begin{array}{c}\text { Prata } \\
\text { Anã }\end{array}$ & \\
\hline Massa & C 1 & $124 \mathrm{c}$ & $119 \mathrm{c}$ & $213 a$ & $182 \mathrm{~b}$ & $164 \mathrm{~b}$ & $117 \mathrm{c}$ & 14,8 \\
\hline média & $\mathrm{C} 2$ & $137 \mathrm{c}$ & $93 \mathrm{~d}$ & $234 \mathrm{a}$ & $177 b$ & $169 \mathrm{~b}$ & $140 \mathrm{c}$ & 12,6 \\
\hline do fruto & $\mathrm{C} 3$ & $133 \mathrm{c}$ & $86 \mathrm{~d}$ & $281 \mathrm{a}$ & $230 \mathrm{~b}$ & $200 \mathrm{~b}$ & $116 \mathrm{~cd}$ & 20,6 \\
\hline (g) & $\mathrm{C} 4$ & $157 \mathrm{~b}$ & $96 \mathrm{c}$ & $300 \mathrm{a}$ & $172 \mathrm{~b}$ & $157 \mathrm{~b}$ & & 20,8 \\
\hline Massa & C 1 & $140 \mathrm{c}$ & $119 \mathrm{~cd}$ & $234 \mathrm{a}$ & $194 \mathrm{~b}$ & $187 \mathrm{~b}$ & $127 \mathrm{~cd}$ & 18,6 \\
\hline fruto & $\mathrm{C} 2$ & $149 \mathrm{c}$ & $97 d$ & $253 a$ & $202 \mathrm{~b}$ & $192 \mathrm{~b}$ & $156 \mathrm{c}$ & 18,2 \\
\hline central & $\mathrm{C} 3$ & $146 \mathrm{c}$ & $86 \mathrm{~d}$ & $321 \mathrm{a}$ & $259 \mathrm{~b}$ & $233 \mathrm{~b}$ & $129 \mathrm{~cd}$ & 21,3 \\
\hline (g) & $\mathrm{C} 4$ & $172 b$ & $102 \mathrm{c}$ & $335 \mathrm{a}$ & $194 \mathrm{~b}$ & $177 b$ & & 20,0 \\
\hline Compr. & C 1 & $14,2 \mathrm{c}$ & $11,9 d$ & $16,8 \mathrm{a}$ & $16,1 \mathrm{ab}$ & $16,6 \mathrm{~b}$ & $14,2 \mathrm{c}$ & 8,6 \\
\hline fruto & $\mathrm{C} 2$ & $15,6 \mathrm{c}$ & $10,7 \mathrm{~d}$ & 18,3 a & $17,3 \mathrm{ab}$ & $16,5 \mathrm{bc}$ & $15,0 \mathrm{c}$ & 10,6 \\
\hline central & $\mathrm{C} 3$ & $16,2 \mathrm{c}$ & $11,0 \mathrm{e}$ & 21,1 a & $19,5 \mathrm{~b}$ & $18,6 \mathrm{~b}$ & $14,4 \mathrm{~d}$ & 9,2 \\
\hline$(\mathrm{cm})$ & $\mathrm{C} 4$ & $16,6 \mathrm{c}$ & $12,0 \mathrm{~d}$ & $21,9 \mathrm{a}$ & $18,4 \mathrm{~b}$ & $17,1 \mathrm{bc}$ & & 12,1 \\
\hline Diâm. & C 1 & $3,4 \mathrm{c}$ & $3,5 \mathrm{bc}$ & $4,0 \mathrm{a}$ & $3,7 \mathrm{ab}$ & $3,7 \mathrm{ab}$ & $3,5 \mathrm{c}$ & 7,6 \\
\hline fruto & $\mathrm{C} 2$ & $3,4 \mathrm{c}$ & $3,4 \mathrm{c}$ & $4,0 \mathrm{a}$ & $3,7 \mathrm{~b}$ & $3,7 \mathrm{~b}$ & $3,8 \mathrm{~b}$ & 7,0 \\
\hline central & $\mathrm{C} 3$ & $3,3 \mathrm{c}$ & $3,3 \mathrm{c}$ & $4,5 \mathrm{a}$ & $4,2 \mathrm{ab}$ & $4,0 \mathrm{~b}$ & $3,5 \mathrm{c}$ & 11,6 \\
\hline$(\mathrm{cm})$ & $\mathrm{C} 4$ & $3,5 \mathrm{~b}$ & $3,5 \mathrm{~b}$ & $4,6 \mathrm{a}$ & $3,8 \mathrm{~b}$ & $0,6 \mathrm{~b}$ & & 8,6 \\
\hline Espes. & C 1 & $0,32 \mathrm{bc}$ & $0,29 \mathrm{c}$ & 0,38 a & $0,38 \mathrm{a}$ & $0,35 \mathrm{ab}$ & $0,30 \mathrm{c}$ & 15,7 \\
\hline casca & $\mathrm{C} 2$ & $0,32 \mathrm{ab}$ & $0,29 b$ & 0,37 a & $0,32 \mathrm{a}$ & $0,37 \mathrm{a}$ & $0,32 \mathrm{ab}$ & 20,5 \\
\hline fr. central & $\mathrm{C} 3$ & $0,30 \mathrm{~cd}$ & $0,26 \mathrm{~d}$ & $0,41 \mathrm{ab}$ & $0,38 \mathrm{ab}$ & $0,33 \mathrm{bc}$ & $0,28 \mathrm{~d}$ & 16,5 \\
\hline$(\mathrm{cm})$ & $\mathrm{C} 4$ & $0,36 \mathrm{~b}$ & $0,24 \mathrm{c}$ & $0,42 \mathrm{a}$ & $0,35 \mathrm{~b}$ & $0,35 \mathrm{~b}$ & & 17,2 \\
\hline
\end{tabular}

Médias seguidas pela mesma letra na linha não diferem entre si, pelo teste de Tukey, a $5 \%$. 
TABELA 5- Massa e número de frutos por penca, das cinco primeiras pencas do cacho, no primeiro (C1), segundo (C2), terceiro (C3) e quarto ciclos (C4), dos genótipos avaliados. Epamig/CTNM, Nova Porteirinha-MG.

\begin{tabular}{|c|c|c|c|c|c|c|c|c|}
\hline & \multirow[b]{2}{*}{ Ciclo } & \multicolumn{6}{|c|}{ Genótipos } & \multirow[b]{2}{*}{$\begin{array}{l}\text { CV } \\
(\%)\end{array}$} \\
\hline & & Pioneira & Caipira & SH3640 & FHIA 1 & FHIA 18 & $\begin{array}{l}\text { Prata } \\
\text { Anã }\end{array}$ & \\
\hline Massa & C 1 & $2,2 \mathrm{c}$ & $3,6 \mathrm{~b}$ & 4,8 a & $4,3 \mathrm{ab}$ & $4,1 \mathrm{ab}$ & $2,1 \mathrm{c}$ & 27,7 \\
\hline penca & $\mathrm{C} 2$ & $2,9 \mathrm{c}$ & $2,4 \mathrm{~cd}$ & $5,5 \mathrm{a}$ & $49, \mathrm{ab}$ & $4,0 \mathrm{~b}$ & $2,5 \mathrm{~cd}$ & 26,9 \\
\hline \multirow[t]{2}{*}{$1(\mathrm{~kg})$} & $\mathrm{C} 3$ & $2,9 \mathrm{~b}$ & $2,3 b$ & 7,0 a & $6,7 \mathrm{a}$ & $5,8 \mathrm{a}$ & $2,4 \mathrm{~b}$ & 33,4 \\
\hline & C4 & $4,1 \mathrm{c}$ & $3,1 \mathrm{~d}$ & 9,1 a & $4,9 \mathrm{bc}$ & $5,3 \mathrm{~b}$ & & 24,2 \\
\hline Massa & C 1 & $2,0 \mathrm{c}$ & $3,0 \mathrm{~b}$ & 3,8 a & 3,8 a & $3,4 \mathrm{ab}$ & $1,9 \mathrm{c}$ & 20,2 \\
\hline penca & C2 2 & $2,5 \mathrm{c}$ & $2,2 \mathrm{c}$ & $4,6 \mathrm{a}$ & $4,1 \mathrm{ab}$ & $3,7 \mathrm{~b}$ & $2,3 \mathrm{c}$ & 21,5 \\
\hline \multirow[t]{2}{*}{$2(\mathrm{~kg})$} & $\mathrm{C} 3$ & $2,9 \mathrm{~b}$ & $2,3 \mathrm{~b}$ & 6,0 a & 5,9 a & $5,4 a$ & $2,1 \mathrm{a}$ & 17,3 \\
\hline & $\mathrm{C} 4$ & $3,1 \mathrm{c}$ & $2,5 \mathrm{c}$ & $6,4 a$ & $4,3 \mathrm{~b}$ & $3,9 \mathrm{~b}$ & & 23,1 \\
\hline Massa & C 1 & $1,6 \mathrm{~d}$ & $2,6 \mathrm{c}$ & 3,6 a & $3,1 \mathrm{~b}$ & $2,6 \mathrm{c}$ & $1,8 \mathrm{~d}$ & 16,2 \\
\hline penca & $\mathrm{C} 2$ & $2,0 \mathrm{c}$ & $2,0 \mathrm{c}$ & $4,3 \mathrm{a}$ & $3,4 \mathrm{~b}$ & $3,2 \mathrm{~b}$ & $2,1 \mathrm{c}$ & 17,1 \\
\hline \multirow[t]{2}{*}{$3(\mathrm{~kg})$} & $\mathrm{C} 3$ & $2,2 \mathrm{c}$ & $2,2 \mathrm{c}$ & $5,4 \mathbf{a}$ & $4,8 \mathrm{ab}$ & $4,4 b$ & $1,9 \mathrm{c}$ & 23,7 \\
\hline & C4 & $2,7 \mathrm{c}$ & $2,5 \mathrm{c}$ & $5,7 a$ & $3,7 \mathrm{~b}$ & $3,3 \mathrm{~b}$ & & 20,7 \\
\hline Massa & C 1 & $1,6 \mathrm{c}$ & $2,4 \mathrm{~b}$ & 3,3 a & $3,0 \mathrm{a}$ & $2,5 \mathrm{~b}$ & $1,7 \mathrm{c}$ & 14,4 \\
\hline penca & C2 & $1,9 \mathrm{c}$ & $1,8 \mathrm{c}$ & 4,1 a & $3,3 \mathrm{~b}$ & $3,1 b$ & $2,1 \mathrm{c}$ & 16,7 \\
\hline \multirow{2}{*}{$4(\mathrm{~kg})$} & C3 & $2,1 \mathrm{c}$ & $1,9 \mathrm{c}$ & 5,1 a & $4,5 \mathrm{ab}$ & $4,1 b$ & $1,8 \mathrm{c}$ & 23,4 \\
\hline & $\mathrm{C} 4$ & $2,5 \mathrm{~cd}$ & $2,3 \mathrm{~d}$ & $5,4 \mathrm{a}$ & $3,5 \mathrm{~b}$ & 3,0 bc & & 21,2 \\
\hline Massa & C 1 & $1,6 \mathrm{~d}$ & $2,3 \mathrm{c}$ & $3,0 \mathrm{a}$ & $2,6 b$ & $2,4 \mathrm{bc}$ & $1,7 \mathrm{~d}$ & 14,5 \\
\hline penca & C2 & $1,8 \mathrm{c}$ & $1,8 \mathrm{c}$ & 3,8 a & $3,1 \mathrm{~b}$ & $2,9 \mathrm{~b}$ & $2,0 \mathrm{c}$ & 16,1 \\
\hline \multirow[t]{2}{*}{$5(\mathrm{~kg})$} & $\mathrm{C} 3$ & $2,0 \mathrm{c}$ & $1,9 \mathrm{c}$ & 4,8 a & $4,3 \mathrm{ab}$ & $3,9 \mathrm{~b}$ & $1,8 \mathrm{c}$ & 22,4 \\
\hline & $\mathrm{C} 4$ & $2,4 \mathrm{~cd}$ & $2,2 \mathrm{~d}$ & 5,1 a & $3,4 \mathrm{~b}$ & $2,9 \mathrm{bc}$ & & 21,1 \\
\hline Número & C 1 & $16 \mathrm{~cd}$ & $31 \mathrm{a}$ & $19 \mathrm{bcd}$ & $20 \mathrm{bc}$ & $22 \mathrm{~b}$ & $16 \mathrm{~cd}$ & 26,2 \\
\hline frutos & $\mathrm{C} 2$ & $19 \mathrm{c}$ & $26 a$ & $21 a b c$ & $24 a b$ & $20 \mathrm{bc}$ & $17 \mathrm{c}$ & 24,6 \\
\hline penca & C3 & $19 \mathrm{~b}$ & $26 a$ & $22 a b$ & $25 \mathrm{a}$ & $25 a$ & $20 \mathrm{~b}$ & 26,1 \\
\hline 1 & C4 & $23 \mathrm{c}$ & $31 \mathrm{a}$ & $26 \mathrm{bc}$ & $26 \mathrm{bc}$ & $30 \mathrm{ab}$ & & 18,2 \\
\hline Número & C 1 & $14 \mathrm{e}$ & $23 a$ & $16 \mathrm{cde}$ & $19 \mathrm{~b}$ & $18 \mathrm{bc}$ & $15 \mathrm{de}$ & 17,5 \\
\hline frutos & C2 & $17 \mathrm{~cd}$ & $23 a$ & $17 \mathrm{~cd}$ & $20 \mathrm{~b}$ & $19 \mathrm{bcd}$ & $15 \mathrm{~d}$ & 16,0 \\
\hline penca & $\mathrm{C} 3$ & $20 \mathrm{bcd}$ & $25 a$ & $19 \mathrm{~cd}$ & $23 a b$ & $23 a b$ & $17 d$ & 19,8 \\
\hline 2 & C4 & $18 \mathrm{c}$ & $25 \mathrm{a}$ & $18 \mathrm{c}$ & $22 \mathrm{~b}$ & $22 b$ & & 17,0 \\
\hline Número & C 1 & $12 d$ & $22 a$ & $15 \mathrm{bc}$ & $16 \mathrm{~b}$ & $15 \mathrm{bc}$ & $14 \mathrm{c}$ & 7,8 \\
\hline frutos & C2 & $14 \mathrm{e}$ & $21 \mathrm{a}$ & $16 \mathrm{~cd}$ & $17 \mathrm{~b}$ & $17 \mathrm{bc}$ & $14 \mathrm{e}$ & 6,9 \\
\hline penca & C3 & $15 \mathrm{~d}$ & $23 a$ & $17 \mathrm{c}$ & $19 \mathrm{~b}$ & $19 \mathrm{~b}$ & $18 \mathrm{~cd}$ & 16,1 \\
\hline 3 & $\mathrm{C} 4$ & $16 \mathrm{~d}$ & $25 \mathrm{a}$ & $17 \mathrm{c}$ & $18 \mathrm{~b}$ & $19 \mathrm{~b}$ & & 7,9 \\
\hline Número & C 1 & $12 \mathrm{c}$ & $20 \mathrm{a}$ & $14 \mathrm{~b}$ & $15 \mathrm{~b}$ & $14 \mathrm{~b}$ & $14 b$ & 8,2 \\
\hline frutos & C2 & $13 c$ & $19 a$ & $16 \mathrm{bc}$ & $17 \mathrm{bc}$ & $16 \mathrm{bc}$ & $14 \mathrm{c}$ & 6,3 \\
\hline penca & C3 & $15 d$ & $21 a$ & $16 \mathrm{c}$ & $18 \mathrm{~b}$ & $18 \mathrm{~b}$ & $16 \mathrm{c}$ & 7,1 \\
\hline 4 & C4 & $15 \mathrm{~d}$ & $23 a$ & $16 \mathrm{c}$ & $18 \mathrm{~b}$ & $18 b$ & & 6,7 \\
\hline Número & C 1 & $12, \mathrm{c}$ & $19 a$ & $14 b$ & $14 b$ & $14 b$ & $14 b$ & 6,5 \\
\hline frutos & $\mathrm{C} 2$ & $13 \mathrm{c}$ & $19 a$ & $15 \mathrm{~b}$ & $16 \mathrm{~b}$ & $16 b$ & $14 \mathrm{c}$ & 6,2 \\
\hline penca & C3 & $14 \mathrm{e}$ & $20 \mathrm{a}$ & $16 \mathrm{c}$ & $17 \mathrm{bc}$ & $18 \mathrm{bc}$ & $15 \mathrm{de}$ & 6,7 \\
\hline 5 & $\mathrm{C} 4$ & $15 \mathrm{~d}$ & $21 a$ & $16 \mathrm{c}$ & $18 \mathrm{~b}$ & $18 b$ & & 7,4 \\
\hline
\end{tabular}

Médias seguidas pela mesma letra na linha não diferem entre si, pelo teste de Tukey, a 5\%.

'FHIA 1' e 'FHIA 18' no segundo e terceiro ciclos; 'Caipira' e 'FHIA 1 ' no quarto ciclo. Portanto, no geral, a 'SH3640' se destacou em massa de frutos por cacho, mas a 'FHIA 1' é que se destacou em número de frutos. Esse resultado se explica pela maior massa média do fruto observada na 'SH3640', nos quatro ciclos avaliados. A maior massa média de fruto foi produzida no quarto ciclo, com $300 \mathrm{~g}$. Esse fruto, exageradamente grande, pode não corresponder às expectativas de um mercado voltado para o consumo de banana 'Prata'. Contrariando as expectativas, a 'Caipira' apresentou maior massa média de fruto no primeiro ciclo e, para 'FHIA 1' e 'FHIA 18', os valores apresentados no quarto ciclo foram inferiores aos do terceiro ciclo.

Com exceção do ‘SH3640’ (não avaliado) e do segundo ciclo da 'Caipira', todos os outros genótipos apresentaram massa de fruto superior à observada por Silva et al. (2000). Todos os genótipos apresentaram, também, valores superiores de massa de frutos por cacho, número de frutos e de pencas por cacho, comprimento e diâmetro do fruto. Isso indica que condições de cultivo mais adequadas, como a irrigação utilizada no presente experimento, podem potencializar a produção desses genótipos. Segundo Moreira (1999), estando o bananal com boa saúde e bom estado nutricional, a produtividade pode aumentar em até $100 \%$, quando se faz a irrigação de modo a suprir efetivamente suas necessidades hídricas. Para Belalcázar Carvajal (1991), o tamanho e a forma do cacho são fatores condicionados geneticamente, porém o número de pencas e frutos pode ser influenciado por condições ambientais adversas que ocorreram na época da diferenciação, como desfolha severa e outros.

Avaliando apenas a massa média do fruto central da mão externa da segunda penca, observou-se a mesma tendência apresentada pela massa média de todos os frutos do cacho (Tabela 4). Entretanto, as médias do primeiro são superiores às do segundo, uma vez que na média da massa de todos os frutos entram os frutos mais leves da extremidade distal do cacho.

A 'SH3640' apresentou maior comprimento do fruto central da mão externa da segunda penca nos quatro ciclos avaliados, igualando-se à 'FHIA 1' nos dois primeiros ciclos. A 'Caipira' apresentou o fruto mais curto. O maior diâmetro do fruto central da mão externa da segunda penca foi dos híbridos 'SH3640', 'FHIA 1' e 'FHIA 18' no primeiro ciclo; ‘SH3640' no segundo; 'SH3640'e 'FHIA 1 ' no terceiro e 'FHIA 18' no quarto ciclo. No geral, 'Caipira' e 'PrataAnã' apresentaram a menor espessura de casca do fruto central da mão externa da segunda penca, igualando-se à 'Pioneira' no primeiro ciclo e à 'SH3640' no segundo e terceiro ciclos. O comprimento e diâmetro de fruto são componentes da massa do cacho e exigências específicas de cada mercado. Já a espessura de casca pode ser um componente de resistência ao transporte, a ser avaliada.

Avaliando as cinco primeiras pencas dos cachos, observouse alto coeficiente de variação para massa (CV de 24,2 a 33,4\%) e 
número de frutos (CV de 18 a 26\%) da primeira penca, indicando grande variação entre os cachos (Tabela 5). A partir da segunda penca, há redução no $\mathrm{CV}$, que fica entre 14 e 22\% para massa e 6 a $19 \%$ para número de frutos. O CV para número de frutos da terceira e quarta pencas são os menores (CV de 6 a 8\%), indicando estabilização. Para Soto Ballestero (2000), as primeiras pencas são mais pesadas que as últimas, porém a primeira é a que apresenta maior heterogeneidade quanto à massa, número de frutos, comprimento e diâmetro dos mesmos. As primeiras pencas apresentam maior número de frutos que as que lhe sucedem.

O híbrido 'SH3640'apresentou a maior massa de pencas para as cinco primeiras pencas do cacho, nos quatro ciclos avaliados, igualando-se à 'FHIA 1' e 'FHIA 18' em alguns casos para pencas um e dois, e à 'FHIA 1' em alguns casos para as pencas três, quatro e cinco (Tabela 5). O maior número de frutos por penca, das cinco primeiras pencas, nos quatro ciclos, foi apresentado pela 'Caipira', igualando-se à 'SH3640', 'FHIA 1' e 'FHIA 18' em alguns casos para pencas um e dois.

Considerando o período compreendido entre o plantio e a colheita do quarto cacho, além da soma da massa desses, e que o espaçamento de plantio utilizado resulta em 1.234 famílias de plantas por ha, calculou-se a produtividade. A maior produtividade foi observada para 'SH3640', 'FHIA 1' e 'FHIA 18' (variando de 70 a 75 t ha $^{-1}$ ano $\left.^{-1}\right)$, seguidos por 'Caipira' e 'Pioneira $\left(41 \mathrm{t} \mathrm{ha}^{-1} \mathrm{ano}^{-1}\right)$. Esses valores são muito superiores à produtividade média de Minas Gerais, que é de 14,6 $\mathrm{t} \mathrm{ha}^{-1}$ ano $^{-1}$ (Agrianual, 2006).

Houve perda de vigor e afloramento de rizomas no quarto ciclo, para 'FHIA 1', 'FHIA 18' e, especialmente, 'SH3640', o mesmo não ocorrendo com 'Caipira', 'Pioneira' e 'Prata-Anã'. Apesar das altas médias de massa de cacho de alguns dos genótipos avaliados, porte médio e tendência de afloramento de rizoma, não houve tombamento ou quebra de plantas.

A incidência da sigatoka pode ter influenciado nos resultados, uma vez que não foi realizado controle. 'Caipira' e 'Pioneira' não apresentaram lesões de sigatoka amarela; os híbridos 'SH3640', 'FHIA 1' e 'FHIA 18' apresentaram mediana incidência; e a 'Prata-Anã' apresentou severa incidência da doença. Em momentos de intensa desfolha promovida pela doença, foi observada queima de frutos pela excessiva exposição ao sol.

\section{CONCLUSÃO}

1) A massa do cacho, de todos os genótipos, superou a da 'Prata-Anã', especialmente para os híbridos 'SH3640', 'FHIA 1' e 'FHIA 18'.

2) A produtividade média de todos os genótipos foi muito superior à produtividade média de banana produzida em Minas Gerais, especialmente os híbridos 'SH3640', 'FHIA 1' e 'FHIA 18'.

\section{REFERÊNCIAS}

AGRIANUAL 2006. Banana. São Paulo: FNP, Consultoria e Agroinformativos, 2005. p. 195-199.

BELALCÁZAR CARVAJAL, S. L. El cultivo del plátano en el trópico. Cali: Impresora Feriva, 1991.367 p.

FAO. FOOD AGRICULTURAL ORGANIZATION. Disponível em: <http://faostat.fao.org/faostat>. Acesso em: 19 jan. 2006.

SILVA, C.R. de R.; SOUTO, R.F., MENEGUCCI, J.L.P.; Propagação da bananeira. Informe Agropecuário, Belo Horizonte, v. 20, n.196, p.16-20, 1999.

MOREIRA, R. S. Banana: teoria e prática de cultivo. São Paulo: Fundação Cargill, 1999. 1 CD-ROM.

SILVA, S. de O. e.; ROCHA, S. A.; ALVES, E. J.; CREDICO, M. di; PASSOS, A. R. Caracterização morfológica e avaliação de cultivares e híbrido de bananeira. Revista Brasileira de Fruticultura, Jaboticabal, v.22, n. 2, p. 161-169. 2000.

SILVA, S. de O. e; RODRIGUES, M. G. V.; REZENDE, J. C. F. de; LIMA NETO, F. P; SILVEIRA, J. R. S. Melhoramento Genético da Bananeira. In: SIMPÓSIO NORTE MINEIRO SOBRE A CULTURA DA BANANA, 1., 2001, Nova Porteirinha. Anais... Montes Claros: Unimontes, 2001. p. 218-237.

SILVA, S. de O. e; PASSOS, A. R.; DONATO, S. L. R.; SALOMÃO, L. C. C.; PEREIRA, L. V.; RODRIGUES, M. G. V.; LIMANETO, F. P.; LIMA, M. B. Avaliação de genótipos de bananeira em diferentes ambientes. Ciência Agrotécnica, Lavras, v. 27, n. 4, p. 737-748, 2003.

SOTO BALLESTERO, M. Bananos: cultivo y comercialización. San José: Imprenta Lil, 2000. 1 CD-ROM. 